BMJ Open Sport \& Exercise Medicine

\title{
Objective assessment of stiffness in the gastrocnemius muscle in patients with symptomatic Achilles tendons
}

\author{
Gafin Morgan (D) , ${ }^{1}$ Rhodri Martin, ${ }^{2}$ Helen Welch, ${ }^{3}$ Lisa Williams, ${ }^{4}$ Keith Morris ${ }^{5}$
}

\begin{abstract}
To cite: Morgan G, Martin R, Welch $\mathrm{H}$, et al. Objective assessment of stiffness in the gastrocnemius muscle in patients with symptomatic Achilles tendons. BMJ Open Sport \& Exercise Medicine 2019;5:e000622. doi:10.1136/ bmjsem-2019-000622
\end{abstract}

Accepted 29 September 2019

Check for updates

(c) Author(s) (or their employer(s)) 2019. Re-use permitted under CC BY-NC. No commercial re-use. See rights and permissions. Published by BMJ

'Podiatry, Cwm Taf Morgannwg University Health Board, Abercynon, UK

${ }^{2}$ Cwm Taf Morgannwg University Health Board, Abercynon, UK

${ }^{3}$ Cwm Taf Morgannwg University Health Board, Abercynon, UK

${ }^{4}$ Orthopaedics, Cwm Taf

Morgannwg University Health

Board, Abercynon, UK

${ }^{5}$ Biomedical Sciences, Cardiff Metropolitan University, Cardiff, UK

Correspondence to Mr Gafin Morgan;

gafin.morgan@wales.nhs.uk

\section{ABSTRACT}

Objectives To establish quantitative values of stiffness for the gastrocnemius muscle in patients diagnosed with Achilles tendinopathy.

Design Case-control study with a single (cross sectional) time point of patients diagnosed with unilateral Achilles tendinopathy and an asymptomatic group.

Methods Sample of 50 participants: 25 participants with symptomatic unilateral Achilles tendinopathy (symptomatic group) with the asymptomatic side of this group used as a control (control group 1). A third group of 25 participants with asymptomatic Achilles tendons (control group 2). Mechanical stiffness parameters, including natural oscillation frequency $(\mathrm{F})$, dynamic stiffness $(\mathrm{S})$, mechanical stress relaxation time (R), logarithmic decrement $(\mathrm{D})$ and creep $(\mathrm{C})$, were assessed using the MyotonPRO. Measurements at the medial and lateral gastrocnemii were taken as non-weight bearing (NWB) and weight bearing (WB).

Results Significant $(p<0.05)$ differences were observed between the mean symptomatic group and control groups 1 and 2 in the WB and NWB conditions for natural oscillation frequency $(F)$, dynamic stiffness (S), and creep (C). There was a significant reduction in S WB and conversely a significant increase in $\mathrm{S}$ of the symptomatic group NWB. Creep (C) and mechanical stress relaxation time $(R)$ were significantly $(p<0.05)$ less in the symptomatic group NWB. Significant differences were observed between the NWB and WB data sets for logarithmic decrement (D). NWB D demonstrated significantly $(p<0.05)$ higher scores than the WB condition. Conclusion This study demonstrated that there was a significant reduction in stiffness of the gastrocnemius muscle in participants with Achilles tendinopathy compared with the two control groups. The WB MyotonPRO measures for the symptomatic groups of $F$ and $S$ were significantly lower, indicating a decrease in the tonal properties $(F)$ and dynamic stiffness $(S)$ of the muscle. The application of MyotonPRO technology to measure changes in stiffness in the gastrocnemius muscle may be useful to measure as part of a rehabilitation programme where specific attention could be placed to increase the tonal properties of the muscle. Further studies are required to develop a robust clinical application of this technology, particularly with regard to Achilles tendinopathy.

\section{INTRODUCTION}

Achilles tendinopathy is a highly prevalent condition observed in athletes and
Key messages

- There was a significant decrease in gastrocnemius muscle tone and dynamic stiffness in the symptomatic Achilles tendon group.

- Weight-bearing (WB) and non-weight-bearing (NWB) measurements demonstrated measurable differences between symptomatic and asymptomatic tendons.

- Significant differences in gastrocnemius stiffness of NWB and WB conditions suggest it is beneficial to measure both conditions.

- The data observed may inform rehabilitation of the gastrocnemius muscle in patients with Achilles tendinopathy.

non-athletes. ${ }^{1}$ It has been defined as a clinical syndrome that is characterised by a combination of pain, diffuse or localised swelling, and impaired performance. ${ }^{23}$ The diagnosis is often made clinically, with treatment aimed at the tendon. Increasingly, however, consideration is being given to the Achilles tendon's related muscle anatomy and how this may influence tendon mechanics, healing and rehabilitation. ${ }^{4}$ The Achilles tendon anatomically connects with the gastrocnemius muscle; therefore, investigating gastrocnemius muscle stiffness may assist in understanding the pathomechanics relating to Achilles tendinopathy and in its rehabilitation.

It is widely accepted that muscle strength is a reliable biomarker for musculoskeletal health. ${ }^{5}$ Previous studies indicate that muscle output in high-force isometric and dynamic muscle actions is positively related to the stiffness of the tendinous structures. This may be due to more effective force transmission from the contractile elements to the bone. ${ }^{6}$ However, the association between the mechanical properties of the connective tissue and contractile muscle output during maximal force exertion is not well understood, particularly with regard to the gastrocnemius muscle. ${ }^{6}$ It has been suggested that the gastrocnemius muscle acts as a spring 
when walking during single support to toe-off of the gait cycle $^{7}$ because the gastrocnemius functioned almost entirely isometrically, that is, maintaining a near constant length, generating minimal power.

Stiffness has been previously suggested as a key mechanical parameter that affects the whole musculotendinous unit, including the function of the tendon, muscle power and locomotive efficiency. ${ }^{8} 9$ Changes to muscle stiffness have been noted in sport-related overuse injuries. ${ }^{10}$ Previous studies of the gastrocnemius muscle in weight-bearing (WB) and non-weight-bearing (NWB) conditions suggest a significant increase in tone and stiffness in the WB condition, with no observed changes in elasticity. ${ }^{11}$ One significant component of stiffness is viscoelasticity, as it assists the clinician in understanding how tissue functions as part of the kinetic system, ${ }^{12}$ that is, slower recovery of muscle/tendon length affecting myofascial force transmission through loss of energy and inefficiency.

The MyotonPRO was used in this study to measure stiffness of the gastrocnemius muscle in vivo in patients diagnosed with Achilles tendinopathy. The MyotonPRO is a handheld device that generates a mechanical oscillation in soft tissues, resulting in a calculation of the mechanical properties of the tissues in vivo. The quantitative mechanical measurements generated by the MyotonPRO provide the clinician with information about the pathological nature of the tissues under question. ${ }^{13}$ Soft tissue measurements taken with the MyotonPRO are captured simultaneously by its accelerometer and can be altered with underlying pathology. ${ }^{8}$ These mechanical properties are components of tissue biomechanics and stiffness, that is, natural oscillation frequency $(\mathrm{F})(\mathrm{Hz})$, Logarithmic Decrement of natural oscillation (D), dynamic stiffness (S) $(\mathrm{N} / \mathrm{m})$, creep ability $(\mathrm{C})$ and mechanical stress relaxation time (R) (ms) (Myoton.com).

The MyotonPRO has been deemed a reliable measure for the mechanical properties of tendon and muscle tissue and provides a quantitative measurement of mechanical soft tissue data. ${ }^{13-16}$ The MyotonPRO has been used to measure the viscoelastic stiffness of muscle by analysing the muscle response to a local mechanical stimulation. ${ }^{14}$ The stiffness of muscle tissue, derived mainly from its viscoelastic properties, can be defined as the resistance of muscle when being passively stretched. ${ }^{17}$ Alamaki et $a l^{18}$ also defined muscle tone as the interaction between viscoelastic properties, structures and neural regulation. Bizzini and Mannion ${ }^{14}$ concluded that the MyotonPRO measures consistent and reliable viscoelastic stiffness of the gastrocnemius muscle. They also concluded that there was a linear relationship with force output, which suggested the MyotonPRO was measuring muscle stiffness rather than subcutaneous tissue. A previous study found that overlying skin of measured tendons has a statistically significant effect on MyotonPRO measurements; however, it did not interfere with the ability of the device to measure physiological variation or trend. ${ }^{19}$ In addition to this, the MyotonPRO was tested for consistency with known material parameters and was shown to provide a very small margin of error.

Comparative technology used to measure soft tissue stiffness includes shear wave elastography; however, this is a measurement of tensile stiffness. In comparison with shear wave elastography, which uses Young's modulus as a surrogate for stiffness, the MyotonPRO generates mechanical compression of anatomy and an oscillation of the tissue, which is used to calculate mechanical transverse stiffness $(\mathrm{N} / \mathrm{m}){ }^{20}$ Comparative measurements of stiffness of the Achilles tendon and gastrocnemius muscle using the MyotonPRO have demonstrated a strong association to Young's modulus measurements of stiffness using shear wave ultrasound elastography. ${ }^{21}$ The MyotonPRO has been shown to have increased intrarater reliability in comparison with shear wave elastography of the gastrocnemius muscle. ${ }^{22}{ }^{20}$ Another commonly used technology that has been shown to provide appropriate elasticity data within muscles is strain ultrasound elastography. ${ }^{23}$ There are many limitations of strain elastography that may provide the MyotonPRO with an advantage with regard to its application. These include the diversity of ultrasound elastography techniques, poor inter-rater and intrarater reliabilities and technical limitations, for example, distance between the transducer and tissue measured. ${ }^{20} 2324$

The null hypothesis proposed that there would be no measurable differences in stiffness between the gastrocnemius muscle in participants with Achilles tendinopathy compared with participants without Achilles tendinopathy.

\section{METHODOLOGY}

This is a case-control study with a single (cross-sectional) time point of patients diagnosed clinically with unilateral Achilles tendinopathy (with symptoms of pain, focal swelling of the tendon and decreased physical function) by the chief investigator and a cohort of participants with normal Achilles tendons. A within-subjects control was used with the asymptomatic side of the symptomatic group. Participant data were age and gender matched. The Morgan et al 2018 protocol was followed.

There were 50 participants in total: 25 patients identified via existing paper-based triage of service user referrals diagnosed with symptomatic unilateral Achilles tendinopathy, that is, the symptomatic group, and 25 participants with asymptomatic Achilles tendons recruited from staff volunteers within the outpatients department via an invitation letter and staff information sheet on local notice boards, that is, control group 2. Data from the asymptomatic side of the symptomatic group was used as control group 1 to provide a within-subjects control. These patients were appointed to a specialist foot and ankle clinic at an outpatient department. The soft tissue mechanical data of the gastrocnemius muscle was collected from each group by the chief investigator using the MyotonPRO and were used to compare each set of data. Reliability testing was carried out on pilot data. 
At the time of the study design, there were no comparative analyses published to use for the power calculation. Therefore, a comparison of between-subjects pilot data collected from clinical activity was used to compare MyotonPRO measurements of the gastrocnemius muscle in symptomatic and asymptomatic Achilles tendons. Based on a simple two-sided t-test and assuming an average symptomatic score of 21 for natural oscillation frequency $(\mathrm{F})(\mathrm{Hz})$, an average asymptomatic score of 29 for $\mathrm{F}$ and a common SD of 8, and assuming a standard $5 \%$ significance threshold, four gastrocnemius measurements (eight in total) were required to demonstrate a statistically significant difference with $90 \%$ power. However, as the pilot data were based on between-subjects data, 25 symptomatic participants were maintained within the trial to ensure adequate sensitivity for a within-subjects control.

MyotonPRO is a class 1 medical device according to European Medical Device Directive (93/42/CEE+2007/47/ $\mathrm{CE})$. There are five steps in the MyotonPRO measurement process, that is, application of prepressure $(0.18 \mathrm{~N})$, a mechanical impulse with quick release $(0.4 \mathrm{~N})$, recording of oscillation $(200 \mathrm{~ms})$, processing of raw signal $(10 \mathrm{~ms})$ and computation of five mechanical parameters $(5 \mathrm{~ms})$. The five mechanical parameters measured were natural oscillation frequency $(\mathrm{F})(\mathrm{Hz})$; logarithmic decrement (D), dynamic stiffness (S) (N/m), creep ability (C) (Deborah number) and mechanical stress relaxation time (R) (ms) (Myoton.com). The mechanical impulse generated by the MyotonPRO does not cause a residual deformation or neurological reaction of the tissue being evaluated. Therefore, repeatability following initial NWB measurement is of no concern for the subsequent WB measurements.

\section{Inclusion and exclusion criteria}

Participants included in the study presented with a unilateral symptomatic Achilles tendinopathy diagnosed clinically with symptoms of focal or generalised swelling and pain of the Achilles tendon, and had an age restriction of 18-70 years to eliminate possible growth variations in younger participants and possible degeneration variation in older participants.

Participants excluded from the trial had a previous surgical repair or a rupture of the Achilles tendon; were prescribed quinolone antibiotics; had a body mass index (BMI) of <35; had a history or symptoms of soft tissue or joint pain below the knee; and had known rheumatological disease, diabetes or connective tissue disease and should not be actively receiving or proceeding through an existing treatment plan for their condition.

\section{Measurement procedure}

Measurements were performed weight bearing (WB) (with the patient standing) and NWB (with the participant lying prone on a couch) with the gastrocnemius muscle exposed and the foot resting at a neutral relaxed position (figure 1).

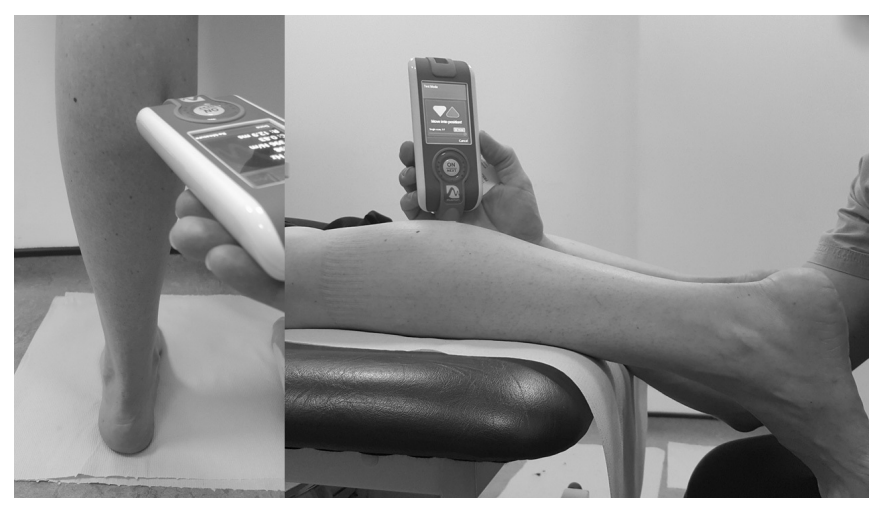

Figure 1 Participant measurement process (left: participant weight bearing; right: participant non-weight bearing/prone).

The MyotonPRO measured (at the centre of each muscle belly) one point at the medial gastrocnemius and one point at the lateral gastrocnemius of both left and right legs. This was repeated for the participant control groups. A multiscan (five measurements) was taken with the MyotonPRO, where the median measurement is taken of each stage. To ensure validity of data and as recommended by Myoton.com, a measurement with a coefficient of variation less than $3 \%$ was accepted, and any measurement above this recommendation was remeasured. Data were imported from the MyotonPRO into Microsoft Excel and analysed using Minitab17 statistical software package and were found to be normally distributed using the Anderson-Darling method. As no significant differences were observed between the medial and lateral gastrocnemius data sets, these were grouped into one data set per group for analysis. Multiple comparisons between MyotonPRO measurement points were compared using the general linear model (GLM) with adjustment for BMI, age and gender. The GLM was used determine the role of factors such as age, BMI and gender to explain any significant variation in outcomes observed by two-sample t-tests. The two-sample t-tests were performed without any adjustments. GLM is an analysis of variance procedure that uses least squares regression to describe the statistical relationship between one or more predictors and a continuous response variable when predictors can be factors and covariates. GLM can perform multiple comparisons between factor level means to find significant differences.

\section{Patient and Public Involvement}

Participants were provided with comprehensive information regarding the research via invitation letter prior to their clinical appointment.

\section{RESULTS}

A two-sample t-test was carried out on each of the WB and NWB gastrocnemius data sets for each group (table 1). Ten t-tests were carried out in total, five for the NWB group and five for the WB group, for each of the MyotonPRO parameters. The MyotonPRO generated results for the five mechanical parameters measured 
Table 1 Reference data of MyotonPRO measures

\begin{tabular}{llllllc}
\hline & & $\begin{array}{l}\text { Frequency } \\
\text { (F) }\end{array}$ & $\begin{array}{l}\text { Decrement } \\
\text { (D) }\end{array}$ & $\begin{array}{l}\text { Stiffness } \\
\text { (S) }\end{array}$ & Creep (C) & $\begin{array}{l}\text { Mechanical stress } \\
\text { relaxation time (R) }\end{array}$ \\
\hline WB symptomatic group & Mean & 21.45 & 1.075 & 496 & 0.806 & 12.19 \\
& SD & 5.4 & 0.278 & 181 & 0.358 & 5.74 \\
\hline WB control group 1 & Mean & 29.6 & 1.086 & 748 & 0.734 & 10.53 \\
\hline WB control group 2 & SD & 16 & 0.483 & 535 & 0.258 & 4.97 \\
\hline & Mean & 30.4 & 1.091 & 741 & 4.7 & 10.82 \\
\hline NWB symptomatic group & SD & 17.6 & 0.42 & 528.9 & 39.3 & 5.2 \\
\hline & SD & 23.41 & 1.324 & 513 & 0.804 & 12.54 \\
\hline NWB control group 1 & Mean & 18.27 & 0.533 & 223 & 0.352 & 5.69 \\
\hline & SD & 3.24 & 0.211 & 74.6 & 0.22 & 3.65 \\
\hline NWB control group 2 & Mean & 18.96 & 1.413 & 351.5 & 1.03 & 16.18 \\
& SD & 3.28 & 0.33 & 74.49 & 0.22 & 3.6 \\
\hline
\end{tabular}

NWB, non-weight-bearing; WB, weight-bearing.

(natural oscillation frequency (F) $(\mathrm{Hz})$, logarithmic decrement of natural oscillation (D), dynamic stiffness (S) $(\mathrm{N} / \mathrm{m})$, creep $(\mathrm{C})$ and mechanical stress relaxation time (R) (ms)) with SE bars.

\section{Natural oscillation frequency (F)}

There were significant $(\mathrm{p}<0.05)$ differences between the mean symptomatic group and mean control group 1 and control group 2 in the WB and NWB conditions for natural oscillation frequency (F) (figure 2). There were greater differences in $\mathrm{F}$ observed in the $\mathrm{WB}$ participants. There was a significant reduction in $\mathrm{F}$ in the symptomatic group WB and a significant increase in $\mathrm{F}$ in the symptomatic group NWB.

\section{Logarithmic decrement (D)}

There were significant $(\mathrm{p}<0.05)$ differences observed between the NWB and WB data sets for logarithmic

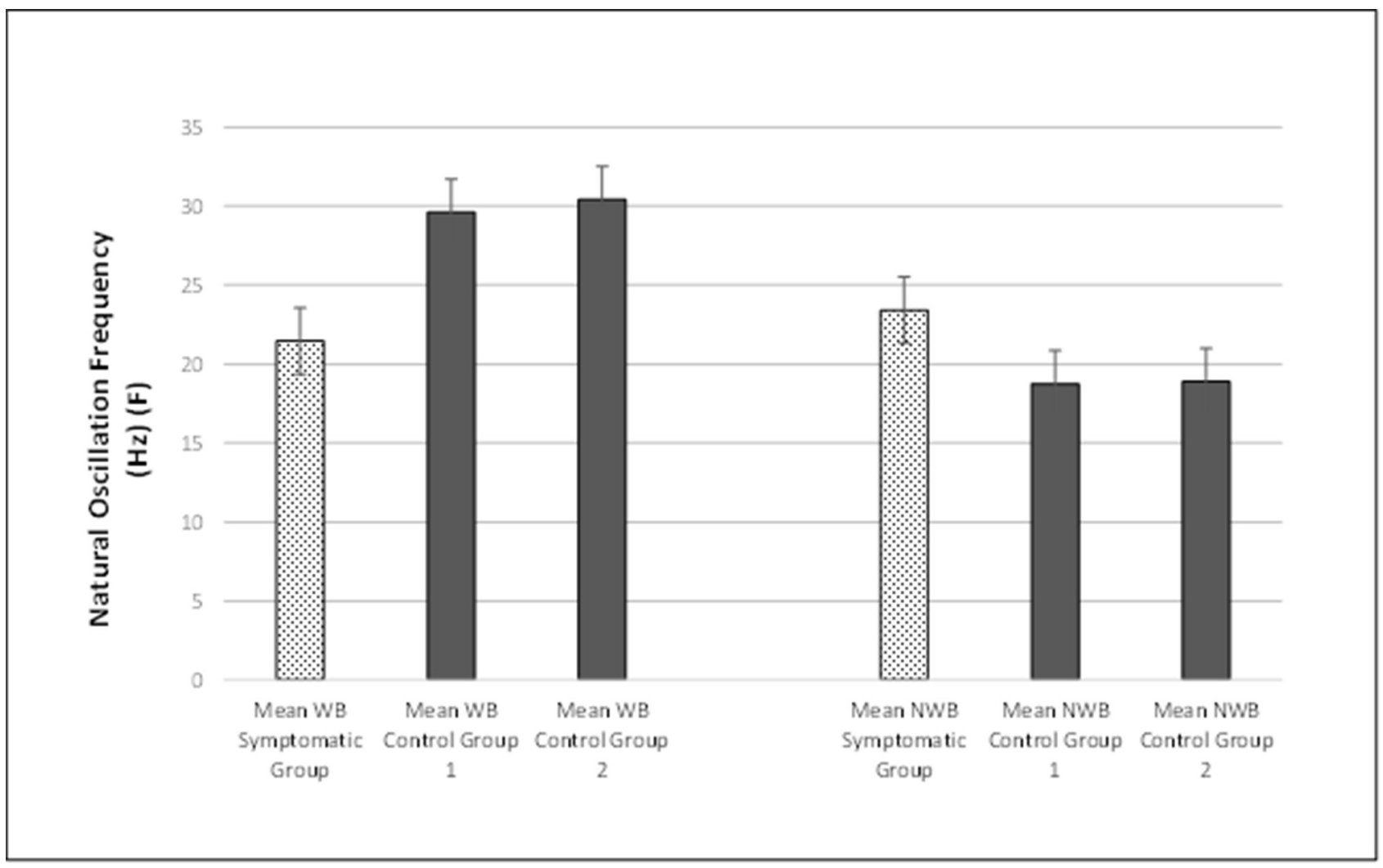

Figure 2 Mean WB and NWB symptomatic groups, control group 1 and control group 2 scores for frequency (F) with SE bars. NWB, non-weight-bearing; WB, weight-bearing. 


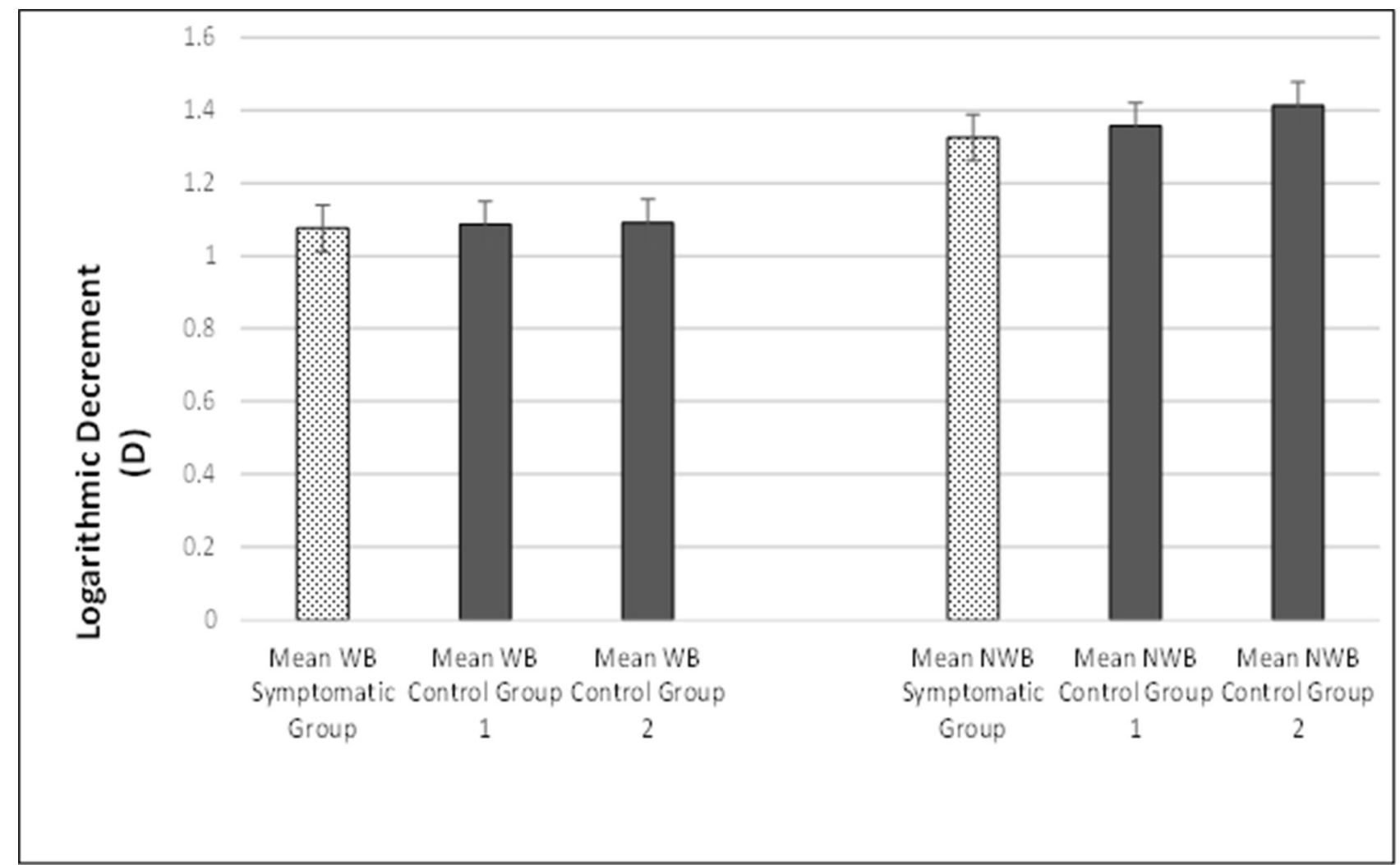

Figure 3 Mean WB and NWB symptomatic groups, control group 1 and control group 2 scores for logarithmic decrement (D) with SE bars. NWB, non-weight-bearing; WB, weight-bearing.

decrement (D) (figure 3). The NWB D group demonstrated significantly $(\mathrm{p}<0.05)$ higher scores than the NWB group overall, representing a reduction in elasticity of the gastrocnemius when NWB.

\section{Dynamic stiffness (S)}

Significant differences $(\mathrm{p}<0.05)$ were observed between the dynamic stiffness (S) of both WB and NWB participants between the mean WB symptomatic group and the mean asymptomatic within-subjects group and asymptomatic control group $(\mathrm{p}<0.05)$ (figure 4$)$. There was a significant $(\mathrm{p}<0.05)$ reduction in $\mathrm{S}$ of the symptomatic group WB and a significant $(\mathrm{p}<0.05)$ increase in $\mathrm{S}$ of the symptomatic group NWB.

\section{Creep (C)}

There were significant $(p<0.05)$ differences between the symptomatic group and the two NWB control groups 1 and two for creep $(\mathrm{C})$ of tissues, with $\mathrm{C}$ being significantly $(p<0.05)$ lower in symptomatic participants for the NWB condition (figure 5).

\section{Mechanical stress relaxation time (R)}

There were significant $(p<0.05)$ differences between the mean NWB symptomatic group and mean NWB control group 1 and control group 2 (figure 6 ). There was a significant $(\mathrm{p}<0.05)$ decrease in $\mathrm{R}$ observed in the symptomatic group.

\section{Control data}

There were no significant $(\mathrm{p}<0.05)$ differences between control group 1 and control group 2.

\section{Effects of age, gender and BMI}

The GLM analysis demonstrated that age and gender affected stiffness values in the symptomatic group only. Demographic data for both groups can be seen in table 2 . The women presented with slightly higher values in the symptomatic group. Age, gender and BMI were adjusted for their expected effects. Gender or age did not significantly affect scores in the control groups. However, age and gender were significant covariates in the symptomatic group. No significant differences $(\mathrm{p}<0.05)$ were observed beween the ages of the participants of the control and symptomatic groups.

\section{DISCUSSION}

This is the first study to measure two points on the gastrocnemius muscle (medially and laterally) with $\mathrm{WB}$ and NWB conditions using the MyotonPRO, in patients with Achilles tendinopathy and two asymptomatic controls. We repeated the methodology of a previous study that investigated the quantitative values for symptomatic and asymptomatic Achilles tendons. ${ }^{25}$ This previous study, however, collected data for the Achilles tendon along eight points of the tendon. This previous methodology ${ }^{25}$ also used a within-subjects control and demonstrated 


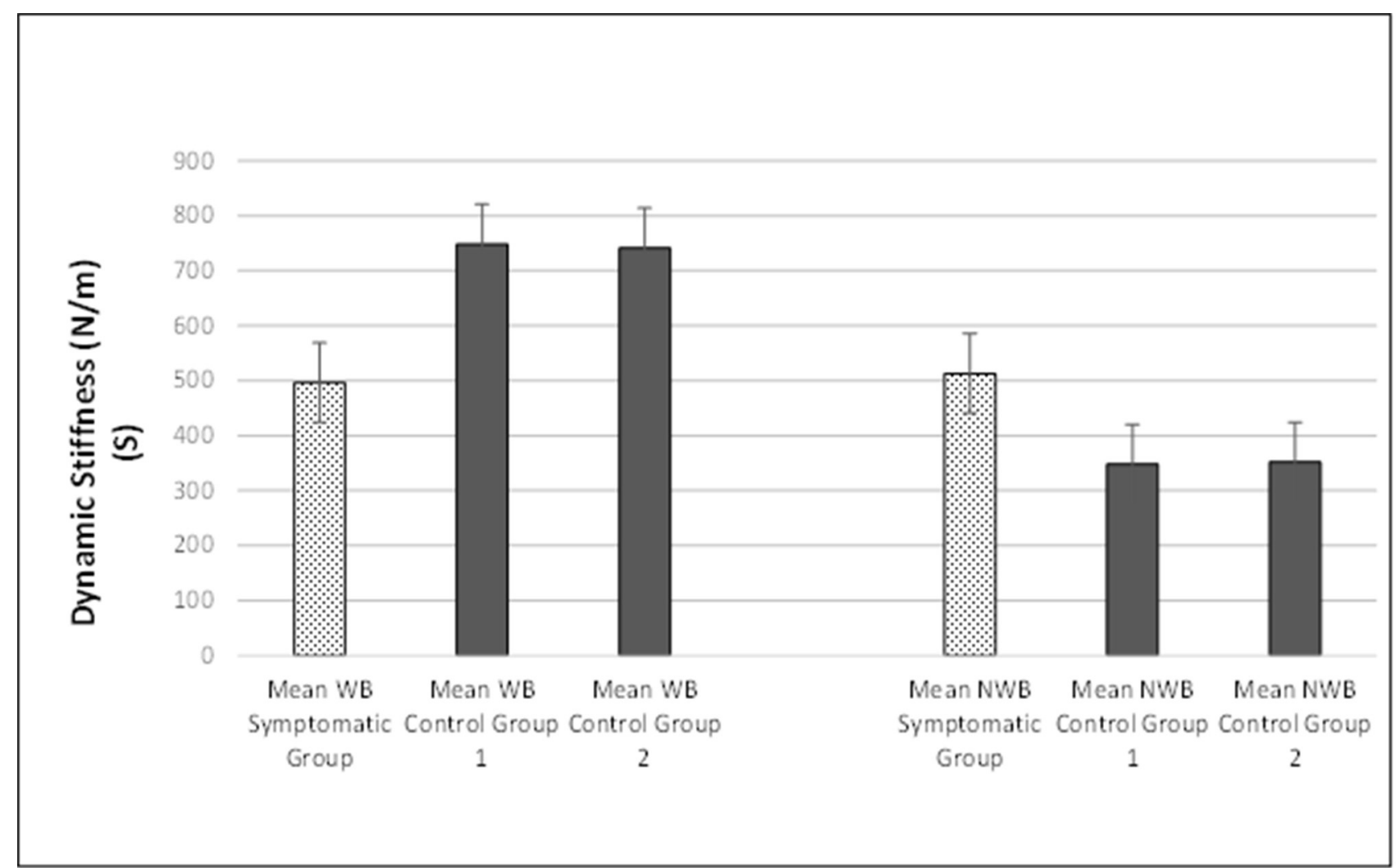

Figure 4 Mean WB and NWB symptomatic groups, control group 1 and control group 2 scores for dynamic stiffness (S) with SE bars. NWB, non-weight-bearing; WB, weight-bearing.

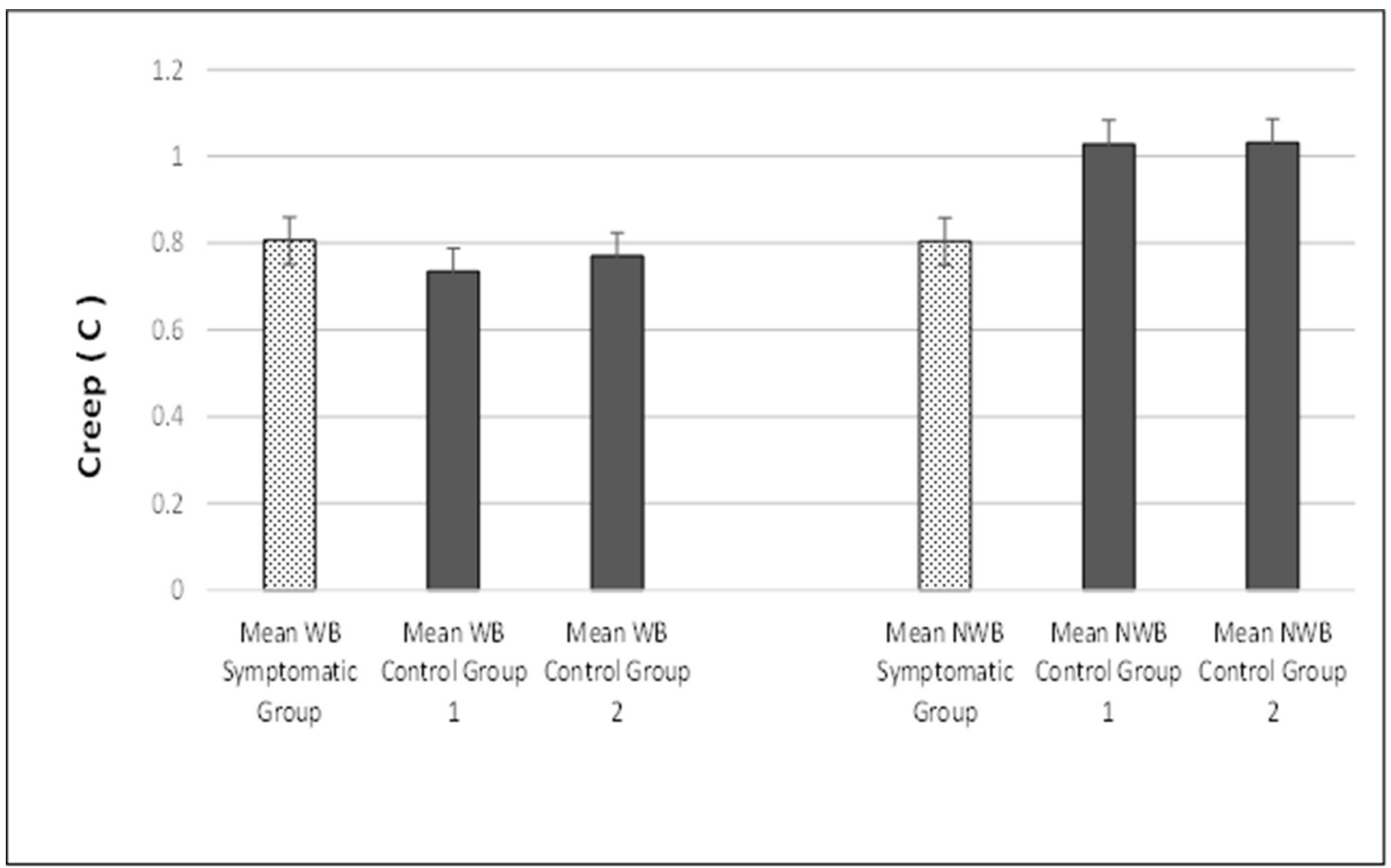

Figure 5 Mean WB and NWB symptomatic groups, control group 1 and control group 2 scores for creep (C) with SE bars. C, creep; NWB, non-weight-bearing; WB, weight-bearing. 


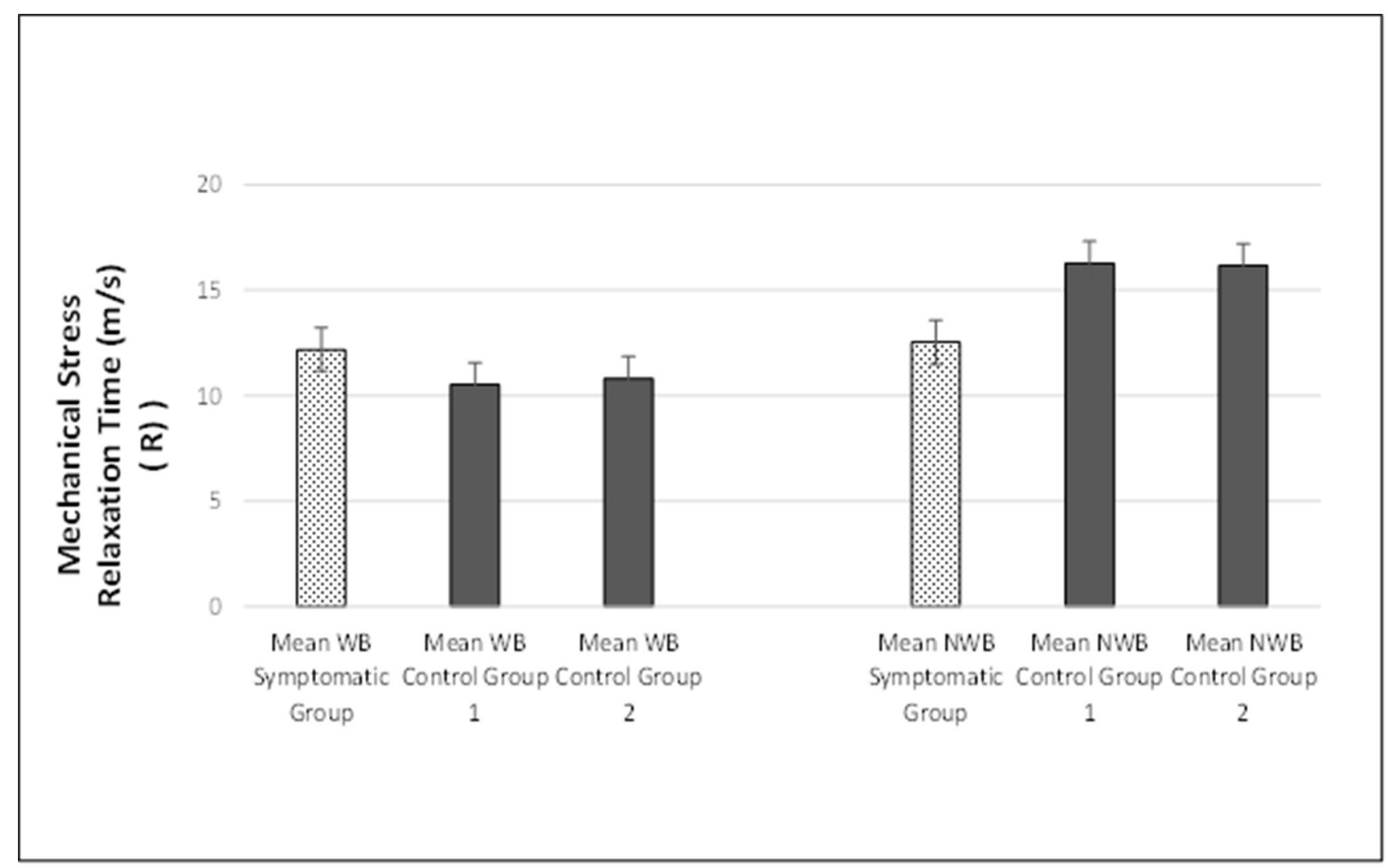

Figure 6 Mean WB and NWB symptomatic groups, control group 1 and control group 2 scores for mechanical stress relaxation time $(\mathrm{R})(\mathrm{m} / \mathrm{s})$ with SE bars. NWB, non-weight-bearing; WB, weight-bearing.

a significant reduction in stiffness of the tendon body correlating with the tendinopathic changes. It has been suggested that the association between the mechanical properties of tendon and muscle tissue is poorly understood. ${ }^{6}$ This study therefore aimed to address this through establishing values of stiffness for the gastrocnemius muscle in patients with Achilles tendinopathy.

The null hypothesis proposed that no differences would be measurable in the gastrocnemius muscle in participants with Achilles tendinopathy compared with participants who did not present with Achilles tendinopathy. The experimental data do not support this hypothesis. This study demonstrated that there were statistically significant differences in the stiffness of the gastrocnemius muscle in participants with Achilles tendinopathy, in particular,

\begin{tabular}{|c|c|c|c|}
\hline & & Control group & Symptomatic group \\
\hline \multirow[t]{2}{*}{$\begin{array}{l}\text { Body mass } \\
\text { index }\end{array}$} & Mean & $\begin{array}{l}25.8 \\
(\min 19.5, \max 33.0)\end{array}$ & $\begin{array}{l}25.5 \\
(\min 19.5, \max 34.3)\end{array}$ \\
\hline & SD & 4.8 & 4 \\
\hline \multirow[t]{2}{*}{ Age } & Mean & $\begin{array}{l}39.6 \\
(\min 26, \max 61)\end{array}$ & $\begin{array}{l}45.2 \\
(\min 5.2, \max 69.0)\end{array}$ \\
\hline & SD & 9.6 & 11.5 \\
\hline \multirow[t]{2}{*}{$m: f$} & Number & $18: 7$ & $17: 8$ \\
\hline & Ratio & 39 & 47 \\
\hline
\end{tabular}

f, female; $m$, male. with the WB condition for the MyotonPRO measures of $\mathrm{F}$ and $\mathrm{S}$ and the NWB condition of $\mathrm{F}, \mathrm{S}, \mathrm{C}$ and R. There was a significant reduction in stiffness of the gastrocnemius muscle in participants within the symptomatic group compared with the two control groups. A previous study investigating the association of postural sway with muscle tone, stiffness and elasticity of the gastrocnemius in healthy participants using Myoton-3 during stable and unstable conditions suggested significantly higher tone and stiffness in the WB gastrocnemius compared with NWB gastrocnemius. ${ }^{11}$ In addition, no changes were observed in elasticity. Vain $e t a l$ also suggested there were no significant differences in stiffness measurements of the gastrocnemius muscle between unstable and stable conditions. This therefore suggests that ballistic muscle action previously observed during postural sway ${ }^{26}$ may not influence muscle stiffness of MyotonPRO measurements. The gastrocnemius muscle has previously been shown to be more active when extended during gait. ${ }^{27}$ Our study therefore suggests that these measurable differences in the gastrocnemius, particularly in the $\mathrm{WB}$ condition, are a result of the Achilles tendinopathy present. Previous studies have found the stiffness of tendons has been shown to determine not only the mechanical properties of the tendon but also the operational range of its muscle fibres, ${ }^{7}$ further supporting a functional relationship between muscle and tendon tissue. This may also be supported by the $\mathrm{WB}$ conditions in the symptomatic group demonstrating a decrease in stiffness. A previous 
study compared the effects of plyometric and isometric training on gastrocnemius muscle and Achilles tendon stiffness, suggesting an increase in muscle stiffness following plyometric training. ${ }^{28}$ This study interestingly did not find changes in gastrocnemius muscle stiffness following isometric training but only following plyometric training. Considering isometric training has been used as a key treatment for Achilles tendinopathy, ${ }^{29}$ this may be only targeting Achilles tendon rehabilitation and not gastrocnemius muscle rehabilitation. It should also be considered that the significant differences observed in the gastrocnemius stiffness between the tendinopathic and control groups in our study may be in relation to corticospinal excitability as suggested in patients with patellar tendinopathy. ${ }^{30}$ It has been suggested that the changes in corticospinal excitability in participants with tendinopathy may result in protective load reducing activity of the relating muscle. ${ }^{30}$

An in vitro study has suggested that tendon viscoelastic stiffness does not significantly modify muscle performance in terms of dynamic response of the muscle. ${ }^{31}$ Our research does not support findings that the elasticity of muscle increases through contraction ${ }^{32}$ in the tendinopathy group, where the $\mathrm{C}$ and $\mathrm{R}$ measurements with the MyotonPRO of the WB condition significantly decreased compared with the NWB condition. The C (creep) measurement indicates the elongation of the tissue over time. ${ }^{33}$ In our study, the WB of $\mathrm{F}$ and $\mathrm{S}$ was significantly lower in the WB conditions, indicating a decrease in the tonal properties (F) and dynamic stiffness (S) of the muscle during the WB condition. Previous studies have suggested an observed increase in dynamic stiffness with passive cyclic stretching, which may correlate with exercise. ${ }^{34}$ Therefore, a benefit of exercise in the management of tendinopathy may include increasing the dynamic stiffness of tendon and muscle tissue. A study of deconditioned healthy men subjected to 20 days of bed rest demonstrated that the stiffness of tendon structures decreased with an increase in hysteresis. ${ }^{35}$ Studies on ageing have also demonstrated an increased tone and stiffness and decreased elasticity with age. ${ }^{5}$ These results suggest that the Achilles tendon was stiffer in subjects with greater muscle stiffness, which may play a role in reducing tendon strain injuries.

Our data suggested that women presented with increased stiffness in the symptomatic group, supporting previous studies suggesting increased differences in stiffness of the gastrocnemius muscle in women. Muraoka et $a \hat{l}^{36}$ suggested that the gender differences observed between soft tissues were mainly due to muscle strength variation between sexes rather than gender.

\section{Limitations}

The limitations of this study are that electromyographical monitoring of the gastrocnemius muscle was not carried out during NWB measurements. This may have added an additional control, ensuring the resting condition of the muscle. Intraday measurements were not carried out, which may have provided data regarding repeatability and intraday variance. The addition of blinding may have added additional validity. There may have been insufficient power in the data to conclude interactions of age, gender and BMI. The exclusion of participants to this trial with a BMI of $<35$ should be noted if applying the results of this study to a wider population.

\section{CONCLUSION}

This study demonstrated that there was a significant reduction in stiffness of the gastrocnemius muscle in participants with Achilles tendinopathy compared with the two control groups. The WB MyotonPRO measures for the symptomatic group of $\mathrm{F}$ and $\mathrm{S}$ were significantly lower, indicating a decrease in the tonal properties $(\mathrm{F})$ and the dynamic stiffness $(\mathrm{S})$ of the muscle. Our data demonstrated that women presented with increased stiffness in the symptomatic group. The application of MyotonPRO technology to measure changes in stiffness in the gastrocnemius muscle may be useful to measure as part of a rehabilitation programme where specific attention could be placed to increase the tonal properties of the muscle. Further studies are required to develop a robust clinical application of this technology, particularly with regard to Achilles tendinopathy.

Acknowledgements The authors thank all the subjects who participated in this research, the Research \& Development Department and all who supported this research project in Cwm Taf Morgannwg University Health Board; J Morgan for proofreading this article and 0 Pearce for data collection.

Contributors GM: chief investigator/lead author, major contribution; RM: consultant physician in sports and exercise medicine, coauthor; HW: physiotherapist, coauthor; LW: consultant orthopaedic surgeon, coauthor; KM: statistician.

Funding This project was supported by Research \& Development at Cwm Taf Morgannwg University Health Board.

\section{Competing interests None declared.}

Patient consent for publication Not required.

Ethics approval Participants were fully informed regarding the nature and aim of the study before signing a written consent form. This study was conducted according to the Helsinki statement and was approved by the local and national ethics committee. Integrated Research Application System Project ID: 155356. Al participants provided signed consent to participate in this study.

Provenance and peer review Not commissioned; externally peer reviewed.

Data availability statement Data are available upon reasonable request.

Open access This is an open access article distributed in accordance with the Creative Commons Attribution Non Commercial (CC BY-NC 4.0) license, which permits others to distribute, remix, adapt, build upon this work non-commercially, and license their derivative works on different terms, provided the original work is properly cited, appropriate credit is given, any changes made indicated, and the use is non-commercial. See: http://creativecommons.org/licenses/by-nc/4.0/.

\section{ORCID iD}

Gafin Morgan http://orcid.org/0000-0001-5483-7365

\section{REFERENCES}

1 McCreesh K, Lewis J. Continuum model of tendon pathology where are we now? Int J Exp Pathol 2013;94:242-7.

2 Alfredson $\mathrm{H}$, Cook J. A treatment algorithm for managing Achilles tendinopathy: new treatment options. Br J Sports Med 2007;41:211-6. 
3 Maffulli N, Wong J, Almekinders LC. Types and epidemiology of tendinopathy. Clin Sports Med 2003;22:675-92.

4 O'Neill S, Watson P. Barry S, Eccentric Exercises For Achilles Tendinopathy Do Not Fully Resolve Plantarflexor Muscle Power Deficits. Br J Sports Med 2014;48:A49-50.

5 Agyapong-Badu S, Warner M, Samuel D, et al. Measurement of ageing effects on muscle tone and mechanical properties of rectus femoris and biceps brachii in healthy males and females using a novel hand-held myometric device. Arch Gerontol Geriatr 2016;62:59-67.

6 Bojsen-Møller J, Magnusson SP, Rasmussen LR, et al. Muscle performance during maximal isometric and dynamic contractions is influenced by the stiffness of the tendinous structures. $J$ Appl Physiol 2005;99:986-94.

7 Fukanga T, Kubo K, Kawakami Y, et al. In vivo behavior of human muscle tendon during walking. procedures of the Royal Society London 2001;B. 268:229-33.

8 Arya S, Kulig K. Tendinopathy alters mechanical and material properties of the Achilles tendon. J Appl Physiol 2010;108:670-5.

9 Peltonen J, Cronin NJ, Stenroth L, et al. Viscoelastic properties of the Achilles tendon in vivo. Springerplus 2013;2:212.

10 Mense S, Simons DG, Pain M. Its nature, diagnosis, and treatment. Philadelphia: Lippincott Williams \& Wilkins, 2001.

11 Vain A, Kums T, Ereline J, et al. Gastrocnemius muscle tone, elasticity, and stiffness in assosciation with postural control characteristics in young men, Profeedings of the Estonian Academy of sciences 2015.

12 Duenwald SE, Vanderby R, Lakes RS. Viscoelastic relaxation and recovery of tendon. Ann Biomed Eng 2009;37:1131-40.

13 Orner S, Kratzer W, Schmidberger J, et al. Quantitative tissue parameters of Achilles tendon and plantar fascia in healthy subjects using a handheld myotonometer. J Bodyw Mov Ther 2017.

14 Bizzini M, Mannion AF. Reliability of a new, hand-held device for assessing skeletal muscle stiffness. Clinical Biomechanics 2003;18:459-61.

15 Lo WLA, Zhao JL, Li L, et al. Relative and absolute interrater Reliabilities of a hand-held Myotonometer to quantify mechanical muscle properties in patients with acute stroke in an inpatient ward. Biomed Res Int 2017;2017:12.

16 Chuang L, Wu C, Lin K, et al. Relative and absolute reliabilities of the myotonometric measurements of hemiparetic arms in patients with stroke, American Congress of rehabilitation medicine. Biomed Res Int 2017;4294028.

17 Schleip R, Naylor IL, Ursu D, et al. Passive muscle stiffness may be influenced by active contractility of intramuscular connective tissue. Med Hypotheses 2006;66:66-71.

18 Alamaki A, Hakkinen A, Ylinen J. Muscle tone in different joint positions and at submaximal isometric torque levels. Physiol Meas 2014.

19 Sohirad S, Wilson D, Waugh C, et al. Feasibility of using a handheld device to characterize tendon tissue biomechanics. PLoS One 2017;12:e0184463.
20 Feng YN, Li YP, Liu CL, et al. Assessing the elastic properties of skeletal muscle and tendon using shearwave ultrasound elastography and MyotonPRO. Sci Rep 2018;8.

21 Niitsu M, Michizaki A, Endo A, et al. Muscle hardness measurement by using ultrasound elastography: a feasibility study. Acta radiol 2011:52:99-105.

22 Kelly JP, Koppenhaver SL, Michener LA, et al. Characterization of tissue stiffness of the infraspinatus, erector spinae, and gastrocnemius muscle using ultrasound shear wave elastography and superficial mechanical deformation. $J$ Electromyogr Kinesiol 2018;38:73-80.

23 Agyapong-Badu S, Aird L, Bailey L, et al. Interrater reliability of muscle tone, stiffness and elastic measurments of rectus femoris and biceps brachii in healthy young and older males, working papers in health sciences 2013;1.

24 Morgan GE, Martin R, Williams L, et al. Objective assessment of stiffness in Achilles tendinopathy: a novel approach using the MyotonPRO. BMJ Open Sport Exerc Med 2018;4:e000446.

25 Loram ID, Maganaris CN, Lakie M. Human postural sway results from frequent, ballistic bias impulses by soleus and gastrocnemius. J Physiol 2005;564:295-311.

26 Reid D, McNair PJ, Johnson S, et al. Electromyographic analysis of an eccentric calf muscle exercise in persons with and without Achilles tendinopathy. Physical Therapy in Sport 2012;13:150-5.

27 Kubo K, Ishigaki T, Ikebukuro T. Effects of plyometric and isometric training on muscle and tendon stiffness in vivo. Physiol Rep 2017;5:e13374.

28 Rio E, Kidgell D, Moseley G, et al. Tendon neuroplastic training: changing the way we think about tendon rehabilitation: a narrative review. Br J Sports Med 2015;0:1-8.

29 Rio E, Kidgell D, Moseley GL, et al. Elevated corticospinal excitability in Patellar tendinopathy compared with other anterior knee pain or no pain. Scand J Med Sci Sports 2016;26:1072-9.

30 Baratta $\mathrm{R}$, Solomonow $\mathrm{M}$. The effect of tendon viscoelastic stiffness on the dynamic performance of isometric muscle. J Biomech 1991;24:109-16.

31 Gavronski G, Veraksitš A, Vasar E, et al. Evaluation of viscoelastic parameters of the skeletal muscles in junior triathletes. Physiol Meas 2007;28:625-37.

32 Solomonow M. Ligaments: a source of musculoskeletal disorders. $J$ Bodyw Mov Ther 2009;13:136-54.

33 Foure A, Nordez A, Cornu C. Effects of plyometric training on passive stiffness of gastrocnemii muscles and Achilles tendon. Eur $J$ Appl Physiol 2011;112:2849.

34 Kubo K, Akima K, Ushiyama J. Effects of 20 days of bed rest on the viscoelastic properties of tendon structures in lower limb muscles. Br J Sports Med 2004;38:324-30.

35 Muraoka T, Muramatsu T, Fukunaga T, et al. Elastic properties of human Achilles tendon are correlated to muscle strength. J Appl Physiol 2005;99:665-9. 